\title{
Optimal Power Factor for the Reactive Load of Small Hydro Power Plants
}

\author{
Angel Tsolov \\ Faculty of Electrical Engineering \\ Technical University of Sofia \\ Sofia, Bulgaria \\ abc@tu-sofia.bg
}

\author{
Boryana Marinova \\ Department of Electrical Power Engineering \\ Technical University of Sofia \\ Sofia, Bulgaria \\ b_marinova@tu-sofia.bg
}

\begin{abstract}
This article explores the operation algorithm of an excitation regulator of synchronous generators of small Hydro Power Plants (HPPs). The aim is to provide optimum economic parameters, consistent with the dynamically changing requirements of electric supply companies. An integral method for maintaining the power factor has been introduced. The proposed method ensures stable operation of generators in different working modes. It is implemented and the economic effect proved significant.
\end{abstract} $A V R$

Keywords-small HPP; power factor; reactive load; excitations;

\section{INTRODUCTION}

The difference between small and large Hydro Power Plants (HPPs) is the electrical network length. Small HPPs are connected to open radial peripheral junctions in the distribution network, where the impedance can be high with a large resistor and little $X / R$ ratio. The production of small HPPs is fragmented, seldom centralized and power generation is dependent on water flow. The hourly, daily and monthly power changes will lead to unobstructed reactive power in both network directions. Therefore, fluctuations in the voltage of the busbars are likely to arise in this network part [1-3]. If these voltage fluctuations are not limited, the power quality requirements delivered to the consumer might be violated. With regard to the work of small-scale generators, connected to a medium voltage grid, a requirement is set. The mode of operation has to restrict the generator's voltage increase above a certain point while generating maximum possible real power [7]. Then the excitation system should ensure a mode of operation maintaining the maximum allowed bus voltage of $20 \mathrm{kV}$ with restriction of maximum generator voltage, maximum and minimum field current [4]. In practice, the structure of excitation regulators (AVR controller) is used (Figure 1). Regulation of excitation is made either by voltage or by power factor (lagging or leading).

\section{1) By voltage}

In this mode, the stator voltage of the generator is regulated with a static characteristic. The stable operation depends on the coefficient of the stasis and its sign.

2) By reactive power
When this mode is in use, the generator works parallel with the network at constant reactive power. This is recommended when the generator works for a long time with load close to the nominal one.

3) By power factor $\cos (\varphi)$

There is a restriction regarding the mode of operation for small generators connected to a medium voltage grid. It should not allow a power factor under certain value - e.g. 0.9 . In this case, an excitation system with constant $\cos (\varphi)$ mode of operation is advisable. The set value should be compliant with the given minimal value.

\section{4) By field current}

This mode could be performed if the regulation is on the field current. The assigned current value changes according to the power line voltage connecting the plant to the power substation. The set value should consequently be adjusted to the maximum voltage value of the $20 \mathrm{kV}$ busbar.

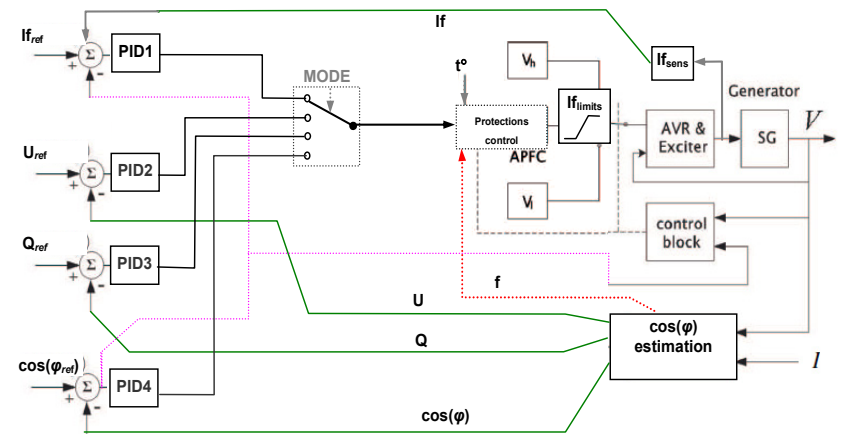

Fig. 1. Excitation regulator (AVR controller) scheme

Assuming that this structure provides reliable operation with a properly-tuned regulative parameter, the question of optimal management, consistent with the economic requirements, remains. The importance of power factor [5] is taken into account. Depending on the registered deviation between the prescribed power factor and the power factor achieved, utility companies apply different criteria and requirements for the mode of operation. In case of deviations of 
$\cos (\varphi)$ from the concurred, fines might follow. At the same time, the static and dynamic regulation stability must be maintained, keeping a mode of operation as close as possible to the manufacturer's requirements - usually they are inconsistent with the regimes required by electrical distribution companies.

Restrictions are introduced on maximum generator voltage, maximal and minimal field current (over excitation ad under excitation), maximum voltage on $20 \mathrm{kV}$ bus bar, overload of the excitation system (thermal protection) and minimum and maximum Real Power of the generator. Concluding that actual maintenance of the desired $\cos (\varphi)$ is possible if the generator is parallel, the active power is above the set threshold, the voltage on the $20 \mathrm{kV}$ bus is below the set limit, the generator voltage is within the allowable range and the excitation current is in the range $\mathrm{I}_{\mathrm{fmin}} \div \mathrm{I}_{\text {fmax }}$. A possible solution for this case is an operation mode with correction by $\cos (\varphi)$. The mode is analogous to the previous one, but it automatically performs a preset parameter $(\cos (\varphi))$ by automatically adjusting the excitation current setting.

\section{MODEL STUDY - RESULTS}

The study of the proposed method of regulation has been examined with a mathematical model in MATLAB/SIMULINK (Figure 2). A detailed description of the model is given in [1]. Corresponding to the actual operating modes research is made for one, and parallel operation of two synchronous generators in small HPP. The schemes for mode of operation are "generator - transformer unit". The results of the research are shown respectively in Figure 3 and Figure 4. Results analysis shows that in this mode of regulation a constant level of the bus voltage is achieved and a good distribution of reactive powers between the units. Implementation in practice shows good results as well. However, long-term maintenance of the required power factor remains a problem.

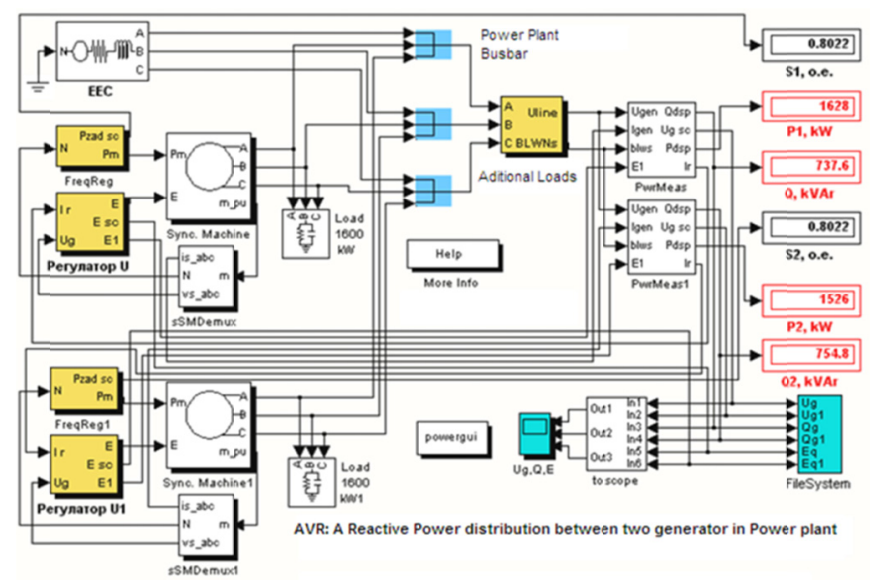

Fig. 2. The SIMULINK model

\section{INTEGRAL POWER FACTOR MAINTENANCE METHODS}

In a 24-hour timespan, maintenance of an optimal power factor $(\cos (\varphi))$ is not always possible. Figure 5 shows the record of voltage deviation, measured from HPP feeders within one day. With high system voltage and regulation by the power factor, limitation - maximum excitation current /thermal limitation/ or maximum generator voltage, is inevitably reached. In order to preserve the stable operation, the system switches to "operation by limit parameters". A solution to the problem is to develop the idea of changing the principle of regulation - impact correction based on an integral method for maintaining the power factor within a certain period of time. The period may be 24-hours, a week, a month, etc, the regulation is implemented according to an integral criterion. This correction allows economically wise regulation of the reactive load. The mode is activated when the basic parameter "optimal $\cos (\varphi)$ " is selected.

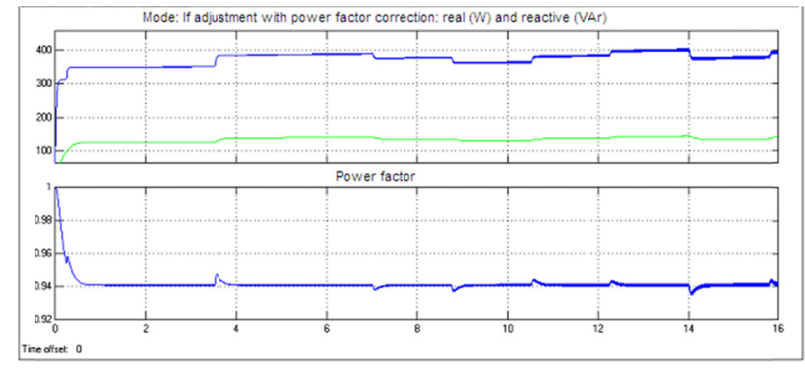

Fig. 3. Operation of one generator

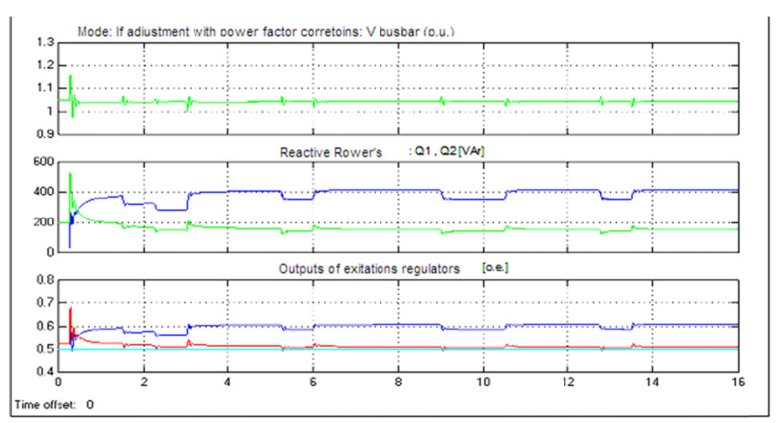

Fig. 4. Operation of two generators in parallel

The introduction of this mode leads to a change in the structure of the regulator - as we see in Figure 6. In this block scheme of the regulator structure, all limitations are integrated into one control block. At the same time, correction of the set parameter is made during the regulation. The corrective function is the current value of $\operatorname{tg}(\varphi)=\frac{P}{Q}$. An additional link "block $\sum \operatorname{tg}(\varphi)$ " is introduced. It indicates that the controller for the excitation system (AVR) accumulates measurements for the changes of $\operatorname{tg}(\varphi)$ with the corresponding sign during the regulation, in any mode of the controller. At the time when there are no restrictions (voltage, temperature, etc.), this accumulated deviation is compensated by adjusting the currently calculated field current value. E.g. when the voltage on the opposite site of the generator is lowered, the field current correction is formed not only by the current value but also by an addition compensating for the accumulated shortage. In this way of control, the change of $\operatorname{tg}(\varphi)$ and the corresponding reactive power (its sign), is compensated in a given period of time - day, month, year. The results from the 
record are shown in Figure 7. The distribution of reactive power is seen within the day. At the beginning of the day, high voltage at the HPP bus bar shows reactive power consumption and a poor power factor. It reaches reactive power, but it is integrated in the time of one day, achieving the optimal distribution of reactive power and power factor.

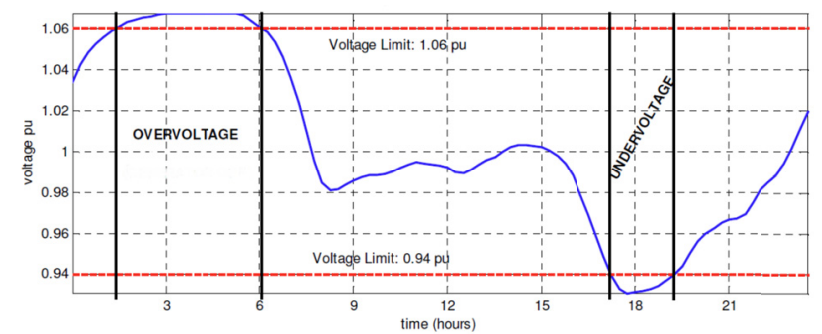

Fig. 5. Record of voltage deviation measured from HPP

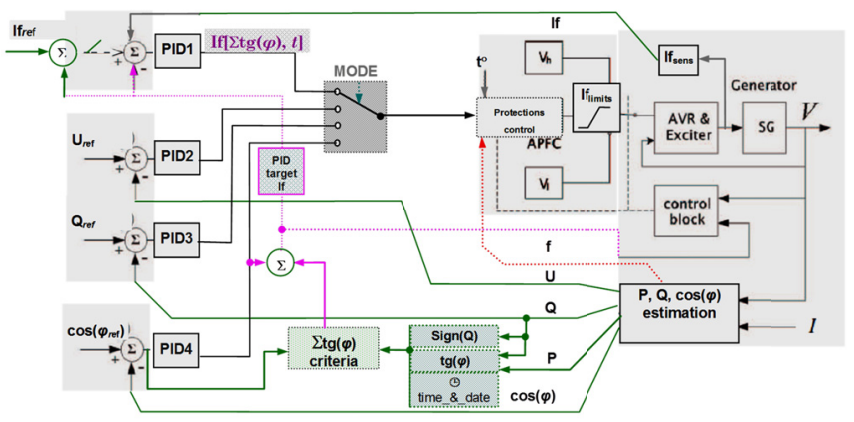

Fig. 6. Alternative regulator structure
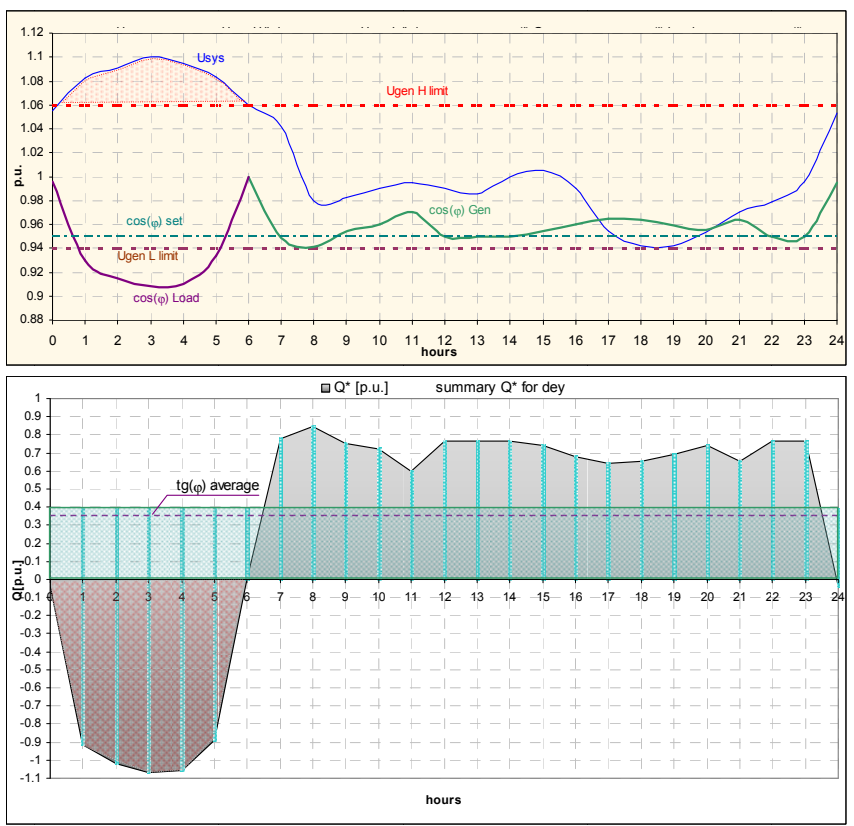

Fig. 7. Results from the alternative structure

\section{CONCLUSIONS}

In power factor correction field current mode, it is ensured that the optimum moment reactive power is generated while ensuring parallel operation of a parallel bus bar. In this mode, AVR works more steadily as the regulation is performed by a primary parameter field current $I_{f}$. Restrictions of regulatory character do not occur. The power distribution is natural. The transient dynamic characteristics are either of an aperiodic nature or of small fluctuations with a decrement of attenuation near one. The implementation of an integral method for maintenance of the power factor gives very positive results. It ensures sustainable operation within a certain time period with optimal economic parameters. An additional advantage of this principle is the possibility of regulation in line with the dynamically changing requirements of electricity supply companies. This method is applied in the excitation control systems for Small HPP "Vlahi" and Small HPP "Garvanitsa". The economic effect is highly positive (no sanctions have been imposed since the introduction of this principle of regulation).

\section{REFERENCES}

[1] D. Tsoneva, A. Tsolov, A. Krumov, "Distribution of the reactive load in synchronous generators an electric power plant under different principles of regulation - mathematical model", Proceedings of the Technical University - Sofia, Vol. 59, No. 1, pp. 151, 2009

[2] T. Katagiri, H. Nakabayashi, Y. Nijo, T. Tamada, T. Noda, N. Hirano, T. Nagata, S. Nagaya, M. Yamane, Y. Ishii, T. Nitta, "Field Test Result of 10MVA/20MJ SMES for Load Fluctuation Compensation, IEEE Transactions on Applied Superconductivity,Vol. 19, No. 3, 2009

[3] D. Dobrilov, A. Tsolov, Means for reactive power compensation - part 2, Proceedings of the Technical University of Sofia, Vol. 64, No. 3, 2014

[4] D. Dobrilov, Means of reactive power compensation-part 1, Proceedings of the Technical University of Sofia, Vol. 64, No. 5, 2013

[5] J. Bjornstedt, O. Samuelsson, "Voltage and Frequency Control for Island Operated Induction Generators", CIRED Seminar: SmartGrids for Distribution, Frankfurt, Germany, June 23-24, 2008

[6] I. Tamrakar, L. B. Shilpakar, B. G. Fernandes, R. Nilsen, "Voltage and frequency control of parallel operated synchronous generator and induction generator with STATCOM in micro hydro scheme", IET Generation, Transmission \& Distribution,Vol. 1, No. 5, pp. 743-750, 2007

[7] D. Dobrilov, Control for small HPP in limited modes, PhD Thesis, 2015

[8] International Electrotechnical Commission, IEC 60034: Rotating Electrical Machines Part 3: Specific Requirements for Turbine-Type Synchronous Machines, 1996

[9] Mathworks, Help for Matlab SimPower System, 2013

[10] P. Kundur, Power Systems Stability and Control, McGraw-Hill, 1994

[11] R. Grondin, I. Kamwa, G. Trudel, L. Gerin-Lajoie, J. Taborda, "Modeling and closed-loop validation of a new PSS concept, the MultiBand PSS", IEEE Power Engineering Society General Meeting, Vol. 3, pp. 1809,2003

[12] H. Wu, G. T. Heydt, "Design of Delayed-Input Wide Area Power System Stabilizer Using the Gain Scheduling Method", IEEE Power Engineering Society General Meeting, Toronto, Canada, Vol. 3, pp. 1709 , July 13-17, 2003 